\title{
Working with guilt and shame
}

\section{Andrew Clark}

\begin{abstract}
SUMMARY
This article describes the psychological experiences of guilt and shame. Although these affects have an important role in holding our communities together, they can also become extremely distressing and debilitating for individuals. As such, they are transdiagnostic problems which are frequently encountered in mental healthcare. The origin of these affects is considered from religious, cultural and psychological perspectives. The article then explores the clinical manifestations of guilt and shame. Finally, consideration is given to therapeutic options, including general approaches as well as specific psychological therapies.
\end{abstract}

\section{DECLARATION OF INTEREST}

None.

The experience of guilt and shame is an essential part of being human. These two affects play an important role in our ability to live together. Without them our families and communities could not survive. However, for many people, intense or chronic feelings of guilt or shame cause great distress.

In the field of mental health, guilt and shame are transdiagnostic problems (Gilbert 2009). They are significant factors in the stories of many people with mental health issues. For some, distressing guilt and shame contribute directly to an ongoing disturbance of mood. For others, the struggle to deal with such intense feelings leads to avoidance strategies which become part of the problem, for example in some people with anxiety disorders, obsessive-compulsive disorders or addictive behaviours. For many, guilt and shame are a consequence of living with a mental illness, particularly in the face of ongoing stigma. Carers of people with mental illness often also struggle with guilt and shame in ways that are often hidden from mental health professionals.

\section{What are guilt and shame?}

Guilt refers to our sense of having done something wrong, either in reality or in our imagination. It relates to real or imagined actions or inactions which have caused real or imagined harm to others. This may then lead to a sense of having to pay a debt or repair something. The word guilt derives from the German word geld which means money or debt. In more severe forms of guilt we may feel we need to be punished. Clinically, the distinction between real and imagined acts is crucial. Someone may feel guilty about something they have actually done or about something they imagine they have done or something they simply thought about. We can also distinguish between guilt that is proportionate to the act ('the punishment fits the crime') and guilt that is out of proportion to what has been done. We will return to the clinical implications of this later.

Shame on the other hand relates to our sense of who we are. We feel that in some way we have fallen short both in our own eyes and in the eyes of others. We then feel a need to hide or conceal an aspect of ourselves. The word shame derives from the Indo-European word skem which means cover. Interestingly, the words chamber and camera (structures that cover or protect) come from the same etymological root.

Guilt and shame often go hand in hand. We might feel guilty about something we have done but ashamed about how this reflects on who we are. However, they can also be experienced independently. For example, one may feel guilty about having passed on one's respiratory infection to someone else without feeling ashamed. One might feel ashamed about an aspect of one's physical appearance without any sense of guilt. A useful way of distinguishing guilt and shame is to consider two different parental responses to their child's misdemeanour. The parent who says 'What you did was naughty!' might induce guilt. The parent who says 'You are a naughty child!' might induce shame.

Guilt is often overt and up front, whereas shame tends to hide itself and so is often harder to identify both for the person suffering and for the person trying to help. Shame seems to have a closer link to the body through our autonomic nervous system, for example blushing. Pines (1987) makes the interesting observation that we have a range of adjectives that describe the spectrum of shame such as bashful, shy, embarrassed, coy, awkward, ashamed, humiliated, mortified. By contrast,

'our language is not nearly so rich in the description of shades of guilt feeling [...] guilt can be seen as being digital rather than analogical in that it represents an either/or and on/off situation that does not offer the same possibilities of shade as does the self-experience of shame. In the eyes of the law a person is either guilty or not guilty.'
Andrew Clark is a consultant psychiatrist in psychotherapy in Bristol working for the Avon and Wiltshire Partnership Mental Health Trust. He also trained as a group analyst at the Institute of Group Analysis. He has a long-standing interest in psychotherapy training for psychiatrists, spirituality in mental health and in the role of forgiveness in the healing process.

Correspondence Andrew Clark, Psychotherapy Service, Cedar House, Blackberry Hill Hospital, Manor Road, Fishponds, Bristol BS16 2EW, UK. Email: andrew. clark@awp.nhs.uk 


\section{The origins of guilt and shame}

\section{Religious perspectives}

The main faith traditions vary significantly in the degree to which they emphasise guilt and shame. Although the Old Testament is a sacred text for all three so-called 'Abrahamic faiths' - Christianity, Judaism and Islam - it is Christianity which has given particular significance to the origin of guilt and shame in the story of Adam and Eve in the Book of Genesis (Box 1). This story is seen as depicting the original 'Fall' of humanity, which in time is redeemed through Christ. Judaism and Islam place less emphasis on the origins of guilt, shame and redemption but rather emphasise practices that can nurture our relationship with God.

Within the Hindu and Buddhist traditions, each individual is viewed as having personal responsibility for actions that lead to guilt. The concept of karma makes provision for all individuals to put right what they have done wrong through the cycle of rebirth. Although both guilt and shame can be seen as a spur to spiritual growth, ultimately the experience of shame would be seen as deriving from a misperception of who we really are.

\section{Cultural perspectives}

Cultural differences, for example in the relative importance given to the individual and the collective, also play a part in fostering guilt or shame. In cultures which emphasise the individual over the collective, guilt and shame would be seen as emotions that protect individuals from each other. By contrast, cultures which emphasise the collective over the individual would see guilt and shame as emotions which protect the community

BOX 1 The story of Adam and Eve

In the Book of Genesis, the first book of the Bible, Adam and Eve are the names given to the first man and woman created by God 'in His own image'. They live in a place of Paradise known as the garden of Eden. God invites them to eat anything from the garden except the fruit of the Tree of the Knowledge of Good and Evil. They live in harmony until one day a serpent entices them to eat the forbidden fruit. As a result they develop self-consciousness and notice that they are naked (their first experience of shame). When God questions them about what they have done, Adam and Eve have their first experience of guilt and in turn seek to pass on the blame (Adam blames Eve and Eve blames the serpent). As a consequence of their action they are banished from the garden. from being shamed. Such cultures may have specific 'honour codes' to reinforce this.

Some cultures or races carry, or are made to carry by other cultures, a collective sense of guilt or shame. Thus, for many hundreds of years the Catholic and Orthodox Churches held the Jewish race as responsible for Christ's crucifixion, leading to centuries of anti-Semitism. More recently, people belonging to a nation that has been responsible for cruelty and violence towards others have felt an ongoing sense of guilt passed down the generations. On a more personal level, some individuals can feel guilt and shame in relation to acts committed by their ancestors.

\section{Psychological perspectives}

Classical psychoanalytic theory paid much more attention to guilt than to shame. In his structural model of the mind, Freud saw guilt as arising from a conflict between the ego and the superego (Freud 1923). For him all guilt was ultimately traceable to Oedipal guilt. Klein, by contrast, related guilt to the developing awareness that one's aggressive impulses might hurt the person one loved - a phase of psychological development she called 'the depressive position' (Klein 1935). Winnicott later described this phase as the stage of concern, emphasising the psychological achievement in reaching this level of awareness (Pedder 1982). Thus the capacity to feel guilt develops with a child's growing awareness that its actions have an impact on others.

Evidence from longitudinal naturalistic observational studies indicates that signs of guilt and attempts to make reparations can be observed in children during their second year (Zahn-Waxler 1992). Writing from a cognitive perspective, Gilbert (2005) describes guilt as an 'affect which warns us if we are close to harming someone or stimulates reparation if we have hurt someone'. He cites research suggesting that the impulse to avoid harming one of one's own species is present in rats and primates. He suggests that in humans this impulse has evolved to encompass a feeling of guilt. Thus guilt 'supports prosocial behaviour and builds interpersonal bonds'. However, an exaggerated sense of responsibility for the welfare of others can then predispose to depression (O'Connor 2002).

Erikson was one of the first psychoanalytic writers to directly address shame. He identified shame as a key issue in the second of eight stages of human development. This stage begins in the child's second year when it starts to 'stand on its own two feet'. Erikson described the essential aspect of this phase as the polarity of 'autonomy 
versus shame and doubt' (Erikson 1950). This fits with the notion that a sense of shame emerges as a child begins to develop a sense of selfconsciousness. In a review of shame and the origin of self-esteem, Jacoby concludes that 'the capacity to experience shame first appears in connection with the realisation that the self can also be seen from the outside' (Jacoby 1994). Shame also became a significant aspect of Kohut's self-psychology. Kohut viewed shame as a collapse of self-esteem or 'narcissistic wound' which is developmentally linked to parental failures to respond attentively and appreciatively to the child as a whole human being (Kohut 1971).

Pines emphasises the important social role of shame and guilt.

\begin{abstract}
'Shame and guilt teach us through painful but inevitable trial and error how to adapt ourselves to social roles and how to influence others to adapt to us. We learn when and how and how much to be open to others: how to manage appropriate closeness and distance: how not to hurt or be hurt: modesty, tact, social sensitivity and sympathy are learnt this way. And we learn to be human by knowing that what we feel others also feel.' (Pines 1995)
\end{abstract}

\section{Problems with guilt: clinical manifestations}

Intense or chronic feelings of guilt are distressing. If the individual has a co-existent mental health problem then they may come to the attention of a mental health professional. In assessing problematic guilt it is useful to distinguish between guilt which is proportionate to whatever the individual has done from guilt which is disproportionate.

\section{Proportionate guilt}

Proportionate guilt might relate to a range of circumstances, for example where the person has harmed or neglected a child. Professionals working with offenders will be familiar with the struggle many have with guilt which is proportionate to their index offence. For some, the weight of this guilt is too much to bear and avoidance strategies come into play such as social withdrawal or drug and alcohol misuse. The clinician is then faced with the difficult judgement of how active they should be in encouraging the individual to face the reality of their guilt. Other offenders struggling with proportionate guilt find comfort in the notion of redemptive acts and actively seek opportunities to atone, if only in part, for their offence.

\section{Disproportionate guilt}

Disproportionate guilt can be subdivided into guilt that is disproportionate to an actual act and guilt that is related to a thought, feeling or imagined act. For example, a mother with depression may feel disproportionately guilty about leaving her child with a babysitter (perhaps because she recalled her own unhappy experiences of being left by her own mother). However, she may also feel guilty about feeling relieved to have some time without her child or having a fleeting thought that she would like to run away from her maternal responsibilities. The question of whether imagining or thinking something harmful is as serious as actually doing it is an interesting moral and philosophical issue. It links with one of Freud's most startling propositions that some criminals commit a crime because unconsciously they already feel guilty about having imagined the crime (Freud 1916). In Freud's formulation, committing the crime ensures that they are punished not only for the act but for thinking the act as well.

Disproportionate guilt is also a common experience for people with obsessive-compulsive disorder. For example, the use of compulsive rituals in an attempt to prevent an imagined event (e.g. harm to a close family member) which would leave the patient feeling guilty.

The most extreme example of disproportionate guilt is seen in patients with depression and delusional guilt. Such patients may be tormented by the belief that they are responsible for a global catastrophe such as an earthquake.

Box 2 lists some of the factors that might contribute to the experience of disproportionate guilt.

\section{Problems with shame: clinical manifestations}

\section{Psychiatric disorders}

Problems with shame may be evident in many psychiatric disorders both as a contributory aetiological factor and as a secondary consequence. Heightened shame sensitivity is often

BOX 2 Factors that might contribute to the experience of disproportionate guilt

- Pre-existing sensitivity to guilt (e.g. through guiltinducing early relationships)

- Reactivation of guilt relating to past action(s)

- Depressed mood

- Obsessional thinking

- External reinforcement by others in the person's current life (e.g. repeated reminders of their responsibility for the harm caused) 
BOX 3 Factors that contribute to heightened sensitivity to feelings of shame

- Shame-inducing early relationships with family and peers

- Physical or sexual abuse

- Bodily disease or disability and cultural attitudes to these

- Reactivation of early experiences of shame

- Low self-esteem

- Depressed mood

- External reinforcement (e.g. repeated reminders of ways in which the individual falls short)

- Membership of a cultural group (large or small) with powerful 'honour codes'

a contributory predisposing factor in disorders that are characterised by low self-esteem (e.g. depression). Many people with anxiety disorders, particularly social phobia, fear what others might see in them. This fear of exposure is usually related to a deep sense of shame. Individuals often develop avoidance strategies that compound the problem. Shame is also a common underlying issue in addictive behaviours. These behaviours may be used to manage shameful feelings. For many people with an addiction, the behaviour itself then becomes a further source of shame leading to a vicious cycle of further addictive behaviour.

\section{Shame in offenders}

Shame is also a key issue in mentally disordered offenders. It is a potent trigger for acts of violence (Gilligan 2000). Some people with heightened shame sensitivity experience a slur or put-down as such a threat to their sense of self that violence towards the source of the slur is felt to be the only way to protect their fragile sense of self. This directly relates to the common preoccupation among such people with the notion of respect. Externally derived respect is seen as critical to managing intense feelings of shame even if these feelings are outside of the individual's awareness. A good example of this is depicted in Ian McEwan's novel Saturday (McEwan 2005). The central character, a neurosurgeon, has a chance confrontation with three men following a minor car accident. During the confrontation, he spots that the leader of these three men has a tremor. Suspecting a neurological disorder he points this out. This is experienced as such a shameful accusation that the leader plots a violent act of revenge against the neurosurgeon and his family.

\section{The body as a source of shame}

The body is a key mediator of shame. The way our body is treated by others, particularly in our early years, contributes significantly to our sensitivity to shame. Thus, many people with an overwhelming sensitivity to shame will have experienced some form of violence to their bodies. Bodily disease and disability, particularly highly visible ones, are also a potent source of shame. This can be partially either mitigated or exacerbated by the response of key attachment figures and by prevailing cultural attitudes towards disease and disability.

Some of the factors contributing to heightened sensitivity to feelings of shame are listed in Box 3.

\section{Clinical illustration}

The following fictional vignette illustrates some of the factors that contribute to heightened feelings of guilt and shame.

Mrs A was a 30-year-old single woman with two children, a girl aged 8 and a boy aged 7 . She became depressed a year ago for what she felt was no apparent reason. She felt particularly guilty that she was unable to be physically affectionate towards her son. This left her feeling ashamed of herself as a mother. This feeling was reinforced by her own mother who was often critical of Mrs A's childcare. In her background history, she spoke of never feeling loved by her mother, who was dependent on alcohol. Mrs A's mother used to blame Mrs A for 'driving her to drink' and often physically beat her. Mrs A felt closer to her father but he was absent from home a lot when she was young. She had a younger brother and the two of them spent a lot of time alone together. During therapy it emerged that when she was 7 years old she and her brother had 'experimented with each other' sexually, having both witnessed their parents having sex on more than one occasion. Following disclosure she was terrified that the therapist would call in the authorities to 'take her away'. Therapeutic work focused on trying to make sense of her longstanding feelings of guilt and shame which had been rekindled a year ago when her children reached the age she and her brother had been at the time of the sexual experimentation. She gradually understood some of the factors which might have led to this and experienced glimpses of compassion for the abused and neglected young girl she was at the time. She also began to have some partial understanding of her mother's emotional problems which might have accounted for her earlier behaviour and for her ongoing criticism of Mrs A. The therapist's nonjudgemental attitude was crucial in allowing her to tell her story. Eventually she was able to have a more affectionate relationship with her own son and to feel better about herself as a mother.

\section{Guilt and shame in carers}

Many carers of people with mental health problems experience distressing levels of guilt and shame. This suffering has for many years been 
hidden from mental health professionals but may now become more apparent with the drive towards greater consideration of the needs of carers. Guilt may relate to beliefs that the carer has contributed to the patient's problem. In cases where the carer is a parent, he or she may believe that they have passed the condition on to their child (which may or may not be true from a genetic point of view). Alternatively, a parent may feel that the way they have raised their child has contributed to the problem. Again, this may or may not have some basis in fact. In these circumstances an important role for the clinician may be to help carers disentangle the sometimes complex issues of responsibility.

Carers, particularly family members, may also struggle with feelings of shame in relation to the person they provide for. For example, they may be concerned about how the patient's illness reflects on them as a parent, spouse, sibling or child, particularly in the face of ongoing stigma towards mental health problems.

\section{Principles of working with guilt and shame}

\section{Therapeutic relationship}

The quality of the therapeutic relationship is of prime importance in working with these experiences. Individuals with mental health problems do not easily share their feelings of guilt, still less their feelings of shame. Indeed, they may not have any words for them. For many, the very act of self-revelation implicit in any therapeutic endeavour feels shameful. For people with marked shame sensitivity this can lead them to 'feel at the mercy of the clinician who, armed with knowledge of their intimate feelings, may be seen as someone who will exact further humiliation' (Cordess 2005). In such circumstances, the clinician's attitude and body language are crucial. It is helpful for the clinician to create an atmosphere of mutual exploration. While respecting the different roles of patient and professional, the clinician needs to bring a sense of shared humanity to the encounter.

\section{Distinguishing guilt and shame}

It is useful to establish the relative contribution of guilt and shame to the patient's distress, bearing in mind that they may not be able to describe shame (patients often use the term 'embarrassed' when they are actually referring to something much more uncomfortable than embarrassment). When exploring guilt it is important to get some sense of whether the guilt is proportionate to whatever they have done. If it is disproportionate then one can begin to unravel the various factors in the patient's life that may be contributing to this. This may take some time but can lead to a more realistic appraisal of their responsibility.

It is also important for the clinician to be sensitive to cultural differences in the experience of guilt and shame. The cultural differences may relate to macrocultures such as membership of individualist or collectivist cultures, or to microcultures such as membership of a street gang.

\section{Treatment of associated psychiatric illness}

Affective disorders are often associated with distressing guilt and shame. Treatment of the mood disturbance, whether through psychological or pharmacological means, often leads to a softening of the intensity of guilt and shame, although the patient may still benefit from some specific therapeutic work addressing these feelings.

In the treatment of obsessive-compulsive disorder, it is not uncommon for patients to partially recover in response to an appropriate behavioural programme but to be left with residual feelings of guilt and shame which may need to be addressed through other approaches, as described below.

By contrast, the acute treatment of psychotic disorders may lead to an increase in guilt and shame as the patient gains insight into what they have done or the implications of their illness. This is particularly relevant in the treatment of patients with severe bipolar disorder, who may feel guilt and shame in relation to their behaviour during a manic episode.

\section{Acceptance and forgiveness}

The two key therapeutic concepts in working with guilt and shame are acceptance and forgiveness.

\section{Acceptance}

An accepting attitude towards the patient promotes the patient's self-acceptance. Acceptance involves a way of being with a patient, warts and all, without needing to make a moral judgement about them. It is a way of being that does not need to jump to conclusions or act precipitously. Overzealous attempts to 'make the patient change' may paradoxically leave them feeling not accepted. Acceptance is communicated by non-verbal cues such as posture and facial expression as well as by verbal ones.

\section{Forgiveness}

Forgiveness is addressed in most mainstream religious faiths, although it is often particularly associated with Christianity. More recently, the concept of forgiveness as a therapeutic process 
${ }^{\dagger}$ For a discussion in Advances see Webster M (2011) Introduction to acceptance and commitment therapy, 17: 309-316. Ed.
BOX 4 Steps that may be important in the process of self-forgiveness

- Acknowledgement of the actual hurt caused - some people have an unrealistic sense of the amount of hurt caused: for example, depressed people tend to exaggerate it, whereas people with antisocial personality disorder may underplay it or not recognise it at all

- Addressing the degree to which one was responsible for this harm - some people feel overly responsible for the actions of others

- Dialogue with the party that was hurt - this happens naturally in many everyday examples of harm to others; in more serious cases, this is not always possible or desirable, although the restorative justice movement has shown that dialogue can facilitate forgiveness (Masters 2004)

- Understanding one's state of mind at the time of the harmful action(s)

- Developing self-compassion

has attracted growing academic interest (Ransley 2004; Worthington 2005). In working with guilt, especially guilt which is proportionate to what the individual has done, we are primarily concerned with self-forgiveness. Like forgiveness of others, this needs to be seen as a process. The steps of this process are listed in Box 4.

\section{Compassion}

For many people the development of selfcompassion is made easier if they have others in their lives who offer them compassion. This allows the possibility of gradually internalising a sense of self-compassion. However, the development of selfcompassion need not depend on the attitudes of others. The growth of mindfulness practice in the mental health field (Mace 2007) has reframed the perennial wisdom gained from meditation practice. Although this practice takes varied forms, one of its core ingredients is the gradual recognition of a part of one's consciousness that observes one's mental processes in a non-judgemental way. This is the beginning of self-compassion.

\section{New directions in psychotherapy}

The general principles of working with guilt and shame mentioned earlier are applicable in psychiatric practice whatever one's specialty. Within the field of psychotherapy, it is interesting to note that traditional models of psychological therapy are being modified to take account of the growing interest in shame. Pines (1995) gives a highly personal account of how his greater awareness of the impact of shame has changed his psychoanalytic practice.

'I have been more able to recognise different shame affects and through this recognition I am more sensitive to the fear of being shamed through therapy ... I am more able, I believe, to stay closer to the material, to maintain dialogue and to enable the patient to build the trust needed to face the shamed aspects of self... The simple fact of speaking to patients openly about shame, or humility, or loss of pride in oneself, may have a very powerful effect with some patients, who thereby feel recognised and accepted by therapists who use these words.'

Group therapy, in particular group analysis, has for some time taken a particular interest in shame and how the therapeutic power of the group can be harnessed to modify people's experience of shame (Smith 2008). Although recognising that groups can sometimes be a negative experience for some people, a well-functioning group can give its members a sense of not being alone with their shameful feelings. This glimpse of the universality of shame can have a significant therapeutic impact.

Within the cognitive-behavioural tradition, Hayes and colleagues (1999) have developed acceptance and commitment therapy. ${ }^{\dagger}$ This makes use of therapeutic strategies such as mindfulness, contact with the present moment, acceptance and cognitive defusion techniques (disidentification from thoughts). More recently, Gilbert (2009) describes a model of therapy known as compassionfocused therapy developed specifically for people with high levels of shame and self-criticism. He refers to it as an integrated therapy that draws on social, developmental, evolutionary and Buddhist psychology, and neuroscience. Central to compassion-focused therapy is compassionate mind training which involves training the patient in the skills and attributes of compassion.

Over the past two decades, mentalisation-based therapy and dialectical behaviour therapy have been specifically developed to treat borderline personality disorder (Linehan 1993; Bateman 2004). Although not specifically targeting problematic guilt and shame, these therapies place particular emphasis on shame-modifying techniques such as empathy and validation.

\section{Guilt and shame in the clinician}

A career in psychiatry inevitably faces one with experiences of guilt (e.g. following the suicide of a patient one has cared for) or shame (e.g. following exam failure). It is important to have access to appropriate support at these times. There may be a need for detailed supervision, self-reflection or even personal therapy if these feelings reach a level which adversely has an impact on one's clinical 
practice (e.g. the guilt-prone psychiatrist who becomes excessively averse to taking appropriate clinical risks).

As a clinician, awareness of one's own experience of guilt and shame can subliminally give the patient a sense that it is safe to share their distress. I'm not advocating widespread self-disclosure, but rather a humble awareness of one's own humanity which in subtle ways can allow the patient to feel accepted.

\section{Acknowledgements}

I am grateful to Judith Rossall, Martin Wells and Alcuin Wilkie for helpful comments on the manuscript.

\section{References}

Bateman A, Fonagy P (2004) Psychotherapy for Borderline Personality Disorder. Oxford University Press.

Cordess C, Davidson K, Morris M, et al (2005) 'Cluster B' antisocial disorders. In Oxford Textbook of Psychotherapy (eds GO Gabbard, J Beck, J Holmes): 269-78. Oxford University Press.

Erikson E (1950) Childhood and Society. Norton.

Freud S (1916) Some character-types met with in psychoanalytic work: Chapter III Criminals from a sense of guilt. Reprinted (in 1953-1974) in Standard Edition of the Complete Psychological Works of Sigmund Freud, vol 14 (trans \& ed J Strachey): 332-3. Hogarth Press.

Freud S (1923) The ego and the id. Reprinted (in 1953-1974) in Standard Edition of the Complete Psychological Works of Sigmund Freud, vol 19 (trans \& ed J Strachey): 3-66. Hogarth Press.

Gilbert P (ed) (2005) Compassion and cruelty: a biopsychosocial approach. In Compassion: Conceptualisations, Research and Use in Psychotherapy: 9-74. Routledge.
Gilbert P (2009) Introducing compassion-focused therapy. Advances in Psychiatric Treatment 15: 199-208.

Gilligan J (2000) Violence. Reflections on our Deadliest Epidemic. Jessica Kingsley Publishers.

Hayes S, Strosahl K, Wilson K (1999) Acceptance and Commitment Therapy: An Experiential Approach to Behaviour Change. Guilford Press. Jacoby M (1994) Shame and the Origins of Self-esteem. Routledge.

Klein M (1935) A contribution to the psychogenesis of manic-depressive states. In The Writings of Melanie Klein (vol 1): 262-89. Hogarth Press.

Kohut H (1971) The Analysis of the Self. International Universities Press.

Linehan M (1993) Cognitive-Behavioural Treatment of Borderline Personality Disorder. Guilford Press.

Mace C (2007) Mindfulness and Mental Health. Brunner-Routledge.

Masters G (2004) Transformation, healing of forgiveness? Assisting victims of crime through restorative practice. In Forgiveness and the Healing Process (eds C Ransley, T Spy): 105-27. Brunner-Routledge.

McEwan I (2005) Saturday. Jonathan Cape.

O'Connor L, Berry J, Weiss J, et al (2002) Guilt, fear, submission and empathy in depression. Journal of Affective Disorders 71: 19-27.

Pedder JR (1982) Failure to mourn, and melancholia. British Journal of Psychiatry 141: 329-37.

Pines M (1987) Shame - what psychoanalysis does and does not say. Group Analysis 20: 16-31.

Pines M (1995) The universality of shame: a psychoanalytic approach. British Journal of Psychotherapy 11: 346-57.

Ransley C, Spy T (2004) Forgiveness and the Healing Process. BrunnerRoutledge.

Smith M (2008) Working with shame in therapeutic groups. Group Analysis 31: 252-64.

Worthington E, O'Connor L, Berry J, et al (2005) Compassion and forgiveness: implications for psychotherapy. In Compassion: Conceptualisations, Research and Use in Psychotherapy (ed P Gilbert): 168-92. Routledge.

Zahn-Waxler C, Radke-Yarrow M, Wagner E, et al (1992) Development of concern for others. Developmental Psychology 28: 126-36.

\section{MCOs}

Select the single best option for each question stem

1 Guilt is:

a synonymous with shame

b always related to an action

c usually pathological

d rare in carers of people with mental health problems

e sometimes transmitted from one generation to another.

\section{Shame is:}

a not related to one's sense of identity

b etymologically related to the word chamber

c not associated with autonomic arousal

$\mathrm{d}$ rarely a consequence of having a disability

e not affected by social stigma.
3 When working with mentally ill offenders:

a guilt is rarely a problem

b redemptive acts may be a useful therapeutic option

c shame is rarely relevant in the trigger for violence

d the psychiatrist's attitude to the patient makes no difference therapeutically

e guilt is never a trigger for further offences.

4 The family of people with mental health problems

a only very rarely feel they have caused their family member's problems

b cannot be helped to feel less guilty about their family member's problems

c may be concerned about how the family member's problems reflect on them $\mathrm{d}$ are never responsible for a family member's problems

e always communicate their suffering to menta health professionals.

5 In working with guilt and shame it is:

a not helpful to distinguish between guilt and shame

b non-verbal communication is not important

c group therapy is rarely useful

d forgiveness may be an important part of the healing process

e mindfulness techniques are unlikely to be beneficial. 